\title{
Assessment of FSD and SDR closures for turbulent flames of alternative fuels
}

\author{
Z.M. Nikolaou • N. Swaminathan
}

Accepted for publication in Flow Turbulence and Combustion on 06/03/2018

\begin{abstract}
Detailed-chemistry DNS studies are becoming more common due to the advent of more powerful modern computer architectures, and as a result more realistic flames can be simulated. Such flames involve many alternative fuels such as syngas and blast furnace gas, which are usually composed of many species and of varying proportions. In this study, we evaluate whether some of the commonly used models for the scalar dissipation rate and flame surface density can be used to model such flames in the LES context. A priori assessments are conducted using DNS data of multi-component fuel turbulent premixed flames. These flames offer unique challenges because of their complex structure having many distinct consumption layers for the different fuel components unlike in a single-component fuel. Some of the models tested showed good agreement with the DNS data and thus they can be used for the multi-component fuel combustion.
\end{abstract}

Keywords DNS · LES · Fractal · Flame surface density · Scalar dissipation rate $\cdot$ alternative fuels

\section{Introduction}

Large Eddy Simulation (LES) of turbulent and reacting flows is challenging and requires accurate but also robust models for sub-grid scale (SGS) turbulence and combustion processes. Despite this, it is gaining popularity in

Z.M. Nikolaou

Affiliation: University of Strathclyde. Present address: Computation-based Science and Technology Research Centre (CaSToRC), The Cyprus Institute, Nicosia, 2121, Cyprus

E-mail: z.nicolaou@cyi.ac.cy

\section{N. Swaminathan}

University of Cambridge

E-mail: ns341@cam.ac.uk 
relevant industries and is likely to become a workhorse in the near future. In turbulent reacting flows, the turbulence influences combustion while the heat release from combustion affects the turbulence and its interaction with scalar mixing and diffusion. These physical processes are therefore interrelated in a non-linear manner as reflected by the nature of the governing equations. Premixed flames, in particular, are important as they are found in many industrial devices and LES modelling for these flames includes various approaches such as Thickened Flames (TF), Flame Surface Density (FSD), G-equation, Unstrained/Strained Flamelets, Conditional Moment Closure etc. and a thorough review on them can be found in [1,2]. The Scalar Dissipation Rate (SDR) $N$ [3, 9], and Flame Surface Density (FSD) $\Sigma$ [4]-7], are two important quantities in turbulent combustion modelling as they appear either directly or indirectly in many of the above modelling approaches. These two quantities are defined as,

$$
N=D\left(\frac{\partial c}{\partial x_{i}} \frac{\partial c}{\partial x_{i}}\right)
$$

and

$$
\Sigma=\sqrt{\frac{\partial c}{\partial x_{i}} \frac{\partial c}{\partial x_{i}}}=\sqrt{N / D}
$$

respectively, where $c$ is a suitably defined progress variable. The diffusion coefficient of the progress variable is $D$ and, if $c$ is defined using temperature then $D=\lambda /\left(\rho c_{p}\right)$ where $\lambda$ is the thermal conductivity of the mixture with a specific heat capacity of $c_{p}$. The SDR appears directly in closures for the reaction rate, $\dot{w}_{c}$, of the progress variable $c$ (e.g. in Bray's model [8]) and it also appears as an unclosed term in the transport equation for the progress variable variance required for Unstrained/Strained Flamelet modelling. The FSD appears in related closures such as in the transport equation for the filtered progress variable $\widetilde{c}$. Many different models have been developed for the SDR and FSD in the past [9-[16. A popular closure for the FSD is to introduce a wrinkling factor $\Xi$ describing the effect of flame front distortion induced by the turbulence. The filtered FSD is then obtained using:

$$
\bar{\Sigma}=\Xi \sqrt{\frac{\partial \bar{c}}{\partial x_{i}} \frac{\partial \bar{c}}{\partial x_{i}}}
$$

where the overbar denotes an LES filtering operation. The wrinkling factor is usually modelled using a fractal-based closure,

$$
\Xi=\left(\frac{\eta_{o}}{\eta_{i}}\right)^{D_{f}-2}
$$

where $\eta_{o}$ and $\eta_{i}$ are the outer and inner cut-off scales respectively and $D_{f}$ is the fractal dimension. Such wrinkling factor-based closures have been used in a number of LES studies of both canonical and practical flames showing overall good results [17]-[25]. The outer cut-off scale $\eta_{o}$ is usually taken to be equal 
to the filter size $\Delta$ in LES. The inner cut-off scale $\eta_{i}$ scales with the Gibson scale $l_{g}=s_{l}^{3} / \epsilon$ in the corrugated flamelets regime, where $s_{l}$ is the laminar flame speed and $\epsilon$ is the dissipation rate of the turbulent kinetic energy [26]. The quantity $\eta_{i}$ scales with the Kolmogorov scale $\eta_{k}$ for the thin reaction zones regime 26. Experimental studies 27] have shown that $\eta_{i}$ scales inversely with the Karlovitz number $K a=\left(\delta_{Z} / \eta_{k}\right)^{2}$, where $\delta_{Z}$ is the Zeldovich flame thickness. Also, other studies 28,29,31 have shown that $\eta_{i}$ scales with $\delta_{Z}$ and the laminar flame thermal thickness $\delta_{l}$ (based on the maximum temperature gradient) for both corrugated and thin reaction zones regimes. The fractal dimension $D_{f}$ depends on the turbulence level and it was parameterised using the ratio $u^{\prime} / s_{l}$ where $u^{\prime}$ is the rms of velocity fluctuations [32. A past study 33. suggested that $D_{f}$ increases from 2 up to a value of $7 / 3$ for increasing $u^{\prime} / s_{l}$, however, larger values were reported 34 for premixed flames.

A large number of models proposed in many past studies for $\Xi$ were investigated using DNS data of premixed combustion with one-step chemistry in [34. It is important to note that Eq. (4) applies in the limit of large filter widths only. DNS studies [35,37] showed that the volume-averaged wrinkling factor follows an exponentially reducing trend for smaller filter width because $\Xi$ should go towards unity in the limit of zero filter width to recover the DNS results. This, prompted the modelling of $\Xi$ as a function of $\Delta^{+}=\Delta / \delta_{l}$ 35. In the same study [35, fractal-based models were proposed for the SDR also with its fractal dimension well above the limit suggested in 33.

A priori assessment 35] using DNS data of a one-step chemistry premixed V-flame showed that the fractal-based models favour the FSD more than the SDR. As a result, an alternative modelling for the sub-grid scale SDR was proposed and validated in [35] by extending an algebraic model derived for RANS in a past study [36]. This SDR model was also extended to account for Le number effects and validated using DNS data with one-step chemistry in 37. This algebraic model also showed an improved agreement with DNS data compared to the traditional linear relaxation model [38. Also, this algebraic model, to be discussed later in Eq. [15), requires the SGS velocity scale, $u_{\Delta}^{\prime}$, and a parameter $\beta_{c}$ to be specified. It was shown that the performance of this model was weakly sensitive to the modelling of $u_{\Delta}^{\prime}$ [37] and that the parameter can be evaluated dynamically [39,40]. This dynamically evaluated SGS model was also used in LES studies 41,42, and gave overall good results. Since the past a priori assessment of this SDR closure used single-step DNS data, its performance was also re-evaluated [43] using DNS data of turbulent premixed $\mathrm{H}_{2}$-air combustion with detailed chemistry [4]. All of these assessments were made for a single-component fuel combustion.

Alternative fuels such as as syngas, blast-furnace gas and coke-oven gas are increasingly being used for power generation in the current energy climate. These gases include many fuel components such as $\mathrm{CO}, \mathrm{H}_{2}$, and $\mathrm{CH}_{4}$ with varying proportions in addition to other components like $\mathrm{CO}_{2}, \mathrm{O}_{2}$ and $\mathrm{N}_{2}$. The relative proportions of these components depend on the production process of these gases, and they typically have lower calorific values in general and thus lower flame speeds. The $\mathrm{H}_{2} \mathrm{O}$ can alter the chemical pathway depending on its 
concentration and it can enhance combustion by supplying $\mathrm{OH}$ radicals 45 , 46, 47. or quench the combustion by absorbing the thermal energy when they are present in copious amount. The $\mathrm{CO}_{2}$ mainly increases the specific heat capacity of the mixture, thus reducing the flame temperature and speed. The $\mathrm{H}_{2}$ can enhance combustion through preferential diffusion effects when it is in large amounts. All of these effects play key roles in determining the flame response to turbulence and despite this, most combustion sub-models currently employed for reacting flow calculations were developed based on DNS of simple fuels as noted earlier.

The distinct feature of the multi-component fuel flame structure is shown in Fig. 1 by plotting the laminar reaction rates and heat release rate, normalised using the respective maximum values, in the progress variable (based on temperature) space. Also, a methane-air flame structure, which is typically used for theoretical analysis and model development in past studies, is shown for comparison. There are many distinct overlapping reaction zones 48 unlike in the single-component fuel, and also when compared to the classical structure expected for one-step reaction (which concentrates all the chemical activities towards the hotter side of the flame). Each of these reaction zones have their own thicknesses which differ quite substantially and even if one defines an overall flame thickness using the temperature gradient, it was observed to be larger compared to the traditional fuel [48. This implies that the turbulent eddies can perturb these structures quite easily and their influences could vary across the flame leading to different levels of distortion to different reaction zones [48. As a result of this, the fractal behaviour and distribution of the heat releasing zone can change and thus it is worthwhile to investigate the commonly used modelling approaches, developed using classical flame structure and simple fuel, for the FSD and SDR. Furthermore, the combustion of these low calorific value fuels might occur in the distributed reaction zones regime of turbulent premixed combustion [48, which would affect the modelling of the SGS reaction rate.

As discussed above, model development and validation has predominantly been done using DNS data of single-component fuels with one-step chemistry, with only occasional use of detailed chemistry. Also, the majority of the experimental data used for the validation are for single-component fuels. Therefore, it is unclear how well the SGS combustion closures developed in past studies would perform for flames of multi-component fuels in light of the foregoing discussion and based on the findings in [48. These alternative fuels are being considered for practical applications for which robust and efficient combustion technologies need to be developed.

Turbulent premixed combustion of multi-component fuel containing a mixture of $\mathrm{CO}, \mathrm{H}_{2}, \mathrm{H}_{2} \mathrm{O}, \mathrm{CH}_{4}, \mathrm{CO}_{2}$ in proportions akin to blast-furnace gas was simulated using DNS with complex chemistry [48]. Varying levels of turbulence were considered for the DNS. Hence, the objectives of this study are to use this DNS database in order to: (a) to assess the performance of some popular FSD and SDR models discussed in section 4. (b) examine the fractal attributes, $D_{f}$, and $\eta_{i}$, for the multi-component fuel flames and their dependence on the 
definitions of the progress variable, and (c) investigate the performance of the algebraic model for the sub-grid scale SDR.

The rest of this paper is organised as follows. Section 2 gives a brief description of the DNS data, the filtering operation and models tested are discussed in sections 3 and 4 respectively. The results are presented in section 5 and the conclusions are summarised in the final section.

\section{DNS databases}

Direct Numerical Simulations were conducted using the SENGA2 code 49] in a past study [48. This code solves the compressible Navier-Stokes equations for reacting flows using a 10th order finite difference scheme for interior points, and a 4th order Runge-Kutta scheme for the time-stepping. These equations using standard notations are,

$$
\begin{gathered}
\frac{\partial \rho}{\partial t}+\frac{\partial \rho u_{k}}{\partial x_{k}}=0 \\
\frac{\partial \rho u_{i}}{\partial t}+\frac{\partial \rho u_{k} u_{i}}{\partial x_{k}}=-\frac{\partial p}{\partial x_{i}}+\frac{\partial \tau_{k i}}{\partial x_{k}} \\
\frac{\partial \rho E}{\partial t}+\frac{\partial \rho u_{k} E}{\partial x_{k}}=-\frac{\partial p u_{k}}{\partial x_{k}}-\frac{\partial q_{k}}{\partial x_{k}}+\frac{\partial \tau_{k m} u_{m}}{\partial x_{k}}, \\
\frac{\partial \rho Y_{\alpha}}{\partial t}+\frac{\partial \rho u_{k} Y_{\alpha}}{\partial x_{k}}=w_{\alpha}-\frac{\partial \rho V_{\alpha, k} Y_{\alpha}}{\partial x_{k}} .
\end{gathered}
$$

where $\alpha$ is the species identifier. Table 1 lists the pertinent details of the DNS data used for this study. These simulations considered premixed flames of a multi-component fuel-air mixture propagating freely in three-dimensional turbulence [48. The composition of the multi-component fuel is listed in Table 2 A detailed chemical mechanism involving 49 reactions and 15 species developed [50,51] specifically for such fuels was used in the DNS. According to the classical combustion diagram proposed by Peters [52] the flames lie in the thin reaction zones regime. Further details such as numerical resolution, boundary conditions, etc. for the simulations are elaborated in [48,53] and thus they are not repeated here.

\section{Filtering operation}

The DNS data are filtered explicitly using a Gaussian filter. The filtered value of a variable $\bar{\phi}(\underline{x}, t)$ is calculated as:

$$
\bar{\phi}(\underline{x}, t)=\int_{\underline{x}^{\prime}=-\infty}^{\infty} G\left(\underline{x}-\underline{x}^{\prime}\right) \phi\left(\underline{x}^{\prime}, t\right) d \underline{x}^{\prime},
$$

where the filter function, $G$, is given by, 


$$
G(\underline{x})=\left(\frac{6}{\pi \Delta^{2}}\right)^{\frac{3}{2}} \exp \left(\frac{-6 \underline{x} \cdot \underline{x}}{\Delta^{2}}\right) .
$$

A box filter can also be used as a filter kernel but the above Gaussian filter is common in many earlier studies and thus it is used here also. The Favre-filtered quantity is defined as,

$$
\widetilde{\phi}(\underline{x}, t)=\frac{\overline{\rho \phi}}{\bar{\rho}}
$$

The DNS data are filtered using normalised filter width ranging from $\Delta^{+}=$ $\Delta / \delta_{l}=0.2$ to 5 . Typical values of $\Delta^{+}$used for high quality LES range from 1 to 3 and thus the higher end of $\Delta^{+}$used for the DNS data are relevant for the large eddy simulations. The DNS data for a period of larger than 1 flame time, defined as $t_{f l}=\delta_{l} / s_{l}$, after reaching a quasi-stationary state are considered for the analysis reported in this study. Conditional averages based on the filtered progress variable $\widetilde{c}$ are also time-averaged over the same period to improve statistical accuracy of the results to be discussed in section 5 .

\section{FSD and SDR models}

The following model was proposed for the FSD wrinkling factor by Dunstan et al. [35],

$$
\Xi=\left[e^{\left(-\theta_{1} \Delta^{+}\right)}+\left(1-e^{\left(-\theta_{2} \Delta^{+}\right)}\right)\left(\frac{\Delta}{\eta_{i}}\right)^{D_{f}-2}\right]
$$

where $\theta_{1}$ and $\theta_{2}$ are model parameters. A model for the filtered SDR was proposed as 35],

$$
\tilde{N}=\Xi \tilde{D} \frac{\partial \tilde{c}}{\partial x_{i}} \frac{\partial \tilde{c}}{\partial x_{i}}
$$

using $\Xi$ given in Eq. 12 but with different values for the model parameters. The values of these parameters used for this study are given in Table 3 for each case and are discussed later in section 5. The letters A, B and $\mathrm{C}$ refer to the three turbulent flames listed in Table 1. It is worth noting that Eq. 13. recovers the DNS result in the limit $\Delta^{+} \rightarrow 0$ because $\Xi$ given in Eq. (12) goes to 1 in this limit. For large enough $\Delta^{+}$, the wrinkling factor follows a power-law in accordance with many past studies. It is implicitly assumed that the wrinkling factor is independent of spatial location and time which allows volume-averaging of the filtered variables in order to provide estimates for the fractal dimension and inner cut-off scale, since for large $\Delta^{+}$one assumes that the variation of the wrinkling factor with filter width is linear on a log-log plot (power-law behaviour).

Due to the importance of the FSD in combustion research, a large number of algebraic models for the FSD wrinkling factor have been developed, and a 
thorough comparison of these models can be found in 34. The best of that lot, based on the test using 1-step chemistry DNS data in 34, is evaluated in this study. This model reads,

$$
\Xi=(1-f)+f\left(\frac{\Gamma u_{\Delta}^{\prime}}{s_{l}}\right)^{\left(D_{f}-2\right)}
$$

where the fractal dimension is evaluated using the heuristic relation of North and Santavica [32, i.e. $D_{f}=2.05 /\left(u_{i n}^{\prime} / s_{l}+1\right)+2.35 /\left(s_{l} / u_{i n}^{\prime}+1\right)$ where $u_{i n}^{\prime}$ is the rms turbulent velocity at the inlet. The function $f$ is given by $f=1 /\left(1+\exp \left[-60\left(\Delta^{+}-1\right)\right]\right)$, and the other function is given by $\Gamma=$ $0.75\left(\Delta / \delta_{Z}\right)^{2 / 3} \exp \left(-1.2\left(u_{\Delta}^{\prime} / s_{l}\right)^{-0.3}\right)$. The DNS result is recovered in the limit of $\Delta^{+} \rightarrow 0$ because $f \rightarrow 0$. This model has been developed by analysing a large set of one-step chemistry DNS datasets and the model constants were evaluated using those datasets only. Hence, it serves as an important benchmark case in order to elucidate it's performance using DNS data of multicomponent fuel with detailed chemistry for the reasons highlighted in section 1 Also, it is important to note that the wrinkling factor given by Eq. (14) depends on the spatial position because of the presence of the sub-grid velocity scale $u_{\Delta}^{\prime}$ and the functions $f$ and $\Gamma$.

Dunstan et al. 35 also proposed an algebraic model for the filtered SDR by extending a RANS model proposed in an earlier study [36] and this LES model is written as,

$$
\widetilde{N}=\widetilde{D} \frac{\partial \widetilde{c}}{\partial x_{i}} \frac{\partial \widetilde{c}}{\partial x_{i}}+\left(1-e^{-\theta_{5} \Delta^{+}}\right)\left[\frac{2 K_{c} s_{l}}{\delta_{l}}+\frac{2 u_{\Delta}^{\prime}}{3 \Delta}\left(C_{3}-\tau C_{4} D a_{\Delta}\right)\right] \frac{\sigma_{c}^{2}}{\beta_{c}}
$$

where $\sigma_{c}^{2}=\widetilde{c^{2}}-\widetilde{c}^{2}$ is the sub-grid variance, and $K_{c}, \beta_{c}, \theta_{5}, C_{i}$ are model parameters defined in 35. The heat-release parameter is $\tau=\left(T_{p}-T_{r}\right) / T_{r}$, where $T_{r}$ and $T_{p}$ are the reactant and product temperatures respectively. The parameter $D a_{\Delta}$ is defined as $D a_{\Delta}=\left(\Delta s_{l}\right) /\left(u_{\Delta}^{\prime} \delta_{l}\right)$. The sub-grid scale turbulent velocity fluctuation is calculated using $u_{\Delta}^{\prime}=\sqrt{\widetilde{u_{i} u_{i}}-\widetilde{u_{i}} \widetilde{u_{i}}} / 3$. The other parameters are given by,

$$
C_{3}=\frac{1.5 \sqrt{K a_{\Delta}}}{1+\sqrt{K a_{\Delta}}}, \quad \text { and } \quad C_{4}=\frac{1.1}{\left(1+K a_{\Delta}\right)^{0.4}}
$$

where $K a_{\Delta}=\left(u_{\Delta}^{\prime} / s_{l}\right)^{3 / 2}\left(\Delta / \delta_{l}\right)^{-1 / 2}$. The sub-grid variance, $\sigma_{c}^{2}$, is an unknown in LES and it is obtained either using an algebraic model or by solving its transport equation. In this study, it is estimated using $\sigma_{c}^{2}=\widetilde{c}(1-\widetilde{c})$. The first term on the right-hand side of Eq. (15) is the resolved field, and the second term represents the unresolved sub-grid part. The exponential term in the sub-grid part ensures that this contribution goes to 0 as $\Delta^{+} \rightarrow 0$ recovering the DNS result. 
A similar SDR model was proposed in [37] to include Lewis number effects and this number is defined as $L e=\lambda /\left(\rho c_{p} D_{k}\right)$ with $D_{k}$ being the diffusivity of the species $k$. This SDR model is,

$$
\widetilde{N}=\widetilde{D} \frac{\partial \widetilde{c}}{\partial x_{i}} \frac{\partial \widetilde{c}}{\partial x_{i}}+\left(1-e^{-\theta_{5} \Delta^{+p}}\right)\left[\frac{2 K_{c} s_{l}}{L e^{1.88} \delta_{l}}+\frac{2 u^{\prime}}{3 \Delta}\left(C_{3}^{\prime}-\tau C_{4}^{\prime} D a_{\Delta}\right)\right] \frac{\sigma_{c}^{2}}{\beta_{c}}
$$

where the definitions of the $C_{i}$ are somewhat different,

$$
C_{3}^{\prime}=\frac{2 \sqrt{K a_{\Delta}}}{1+\sqrt{K a_{\Delta}}} \quad \text { and } \quad C_{4}^{\prime}=\frac{1.2(1-\widetilde{c})^{\phi}}{L e^{2.57}\left(1+K a_{\Delta}\right)^{0.4}}
$$

where $\phi=0.2+1.5|1-L e|$. It is important to note that the definition of the $L e$ for multi-component fuel flames is not quite straightforward. In this study, a mass-fraction averaged $L e$ is used i.e. $L e=\sum Y_{k} L e_{k}$, where $L e_{k}$ is the Lewis number of species $k$ in the multi-component fuel. For the mixture used in this study, $L e=0.98$, and the parameter $p$ is taken to be 1 . The model parameter $\beta_{c}$ is particularly important as it affects the contribution of the sub-grid term. Originally, a constant value of $\beta_{c}=2.4$ was used, but in a later study an empirical expression was proposed which was derived by analysing DNS data of a 1-step chemistry premixed flame [39]. This expression reads,

$$
\beta_{c}=\max \left(\frac{2}{2 c_{m}-1},\left[\frac{1.05 \tau}{(1+\tau)}+0.51\right]^{4.6}\right)
$$

where $c_{m}$ is calculated from a laminar premixed flame 39. This expression for $\beta_{c}$ will be used for both SDR models as given by Eqs. (15) and (17).

It is also important to note that the above two models include parameters which need to be tuned to match the DNS results. In practical LES, this can be done through a dynamic procedure 39,40. The aim of this study is to investigate whether the filtered FSD and SDR can be modelled using the above approaches as a first step.

\section{Results and Discussion}

5.1 Volume-averaged wrinkling factors.

The volume-averaged wrinkling factors are defined as,

$$
\left\langle\Xi_{\Sigma}\right\rangle=\frac{\langle\bar{\Sigma}\rangle}{\left\langle\sqrt{\frac{\partial \bar{c}}{\partial x_{i}} \frac{\partial \bar{c}}{\partial x_{i}}}\right\rangle}
$$

and

$$
\left\langle\Xi_{N}\right\rangle=\frac{\langle\widetilde{N}\rangle}{\left\langle\widetilde{D} \frac{\partial \widetilde{\widetilde{c}}}{\partial x_{i}} \frac{\partial \widetilde{\widetilde{c}}}{\partial x_{i}}\right\rangle}
$$


for the FSD and SDR respectively. The angle brackets denote the volumeaveraging operation. Figures 2 and 3 show the variation of the volume-averaged wrinkling factors for the FSD and SDR for cases A-C. The progress variable $c$ is based on temperature i.e. $c=\left(T-T_{r}\right) /\left(T_{p}-T_{r}\right)$. The symbols denote the DNS data, and the lines are the model predictions using Eq. (12). The fractal dimension is calculated by fitting a best-fit line through the DNS data for large $\Delta^{+}$, and the inner cut-off from the intersection of this fitted line with the $x$-axis. The model parameters given in Table 3 are chosen so that the error between the model and DNS values is minimum.

Firstly, it is important to note that both the FSD and SDR wrinkling factors appear to follow almost a straight line for $\Delta^{+}>1$. The FSD inner cut-off scale is of the order of the laminar flame thickness in accordance with previous studies using one-step chemistry [27,29]. It is also instructive to compare the inner cut-off scale with the Gibson scale $l_{g}$. Assuming Kolmogorov scaling between the largest and smallest scales, $\epsilon$ can be taken to remain constant across these scales. Hence one can estimate the normalised Gibson scale using $l_{g} / \delta_{l}=s_{l}^{3} /\left(\delta_{l} \epsilon\right)=\left(u^{\prime} / s_{l}\right)^{-3}\left(l_{\text {int }} / \delta_{l}\right)$. Using the inlet turbulence parameters listed in Table 1 , one obtains $l_{g} / \delta_{l}=2.2 E-2,9.94 \mathrm{E}-4$, and $2.258 \mathrm{E}-4$ for cases $\mathrm{A}, \mathrm{B}$ and $\mathrm{C}$ respectively. Thus, the Gibson scale is many orders of magnitude smaller than the inner cut-off scale. The inner cut-off scale generally decreases with increase in turbulence level (compare cases A to C in Table 3) as observed in many earlier studies. For cases B and C, they are almost equal due to the higher dissipation rate for case $\mathrm{C}$ which results in a similar flame front wrinkling as for case B.

The SDR inner cut-off scale is also of the order of $\delta_{l}$ (see Table 3) and shows a similar behaviour for cases A-C as the FSD inner cut-off scale. The FSD fractal dimensions for cases A and B are almost the same, despite the higher turbulence level in case B, while the FSD fractal dimension for case $\mathrm{C}$ is larger than both $\mathrm{A}$ and $\mathrm{B}$ denoting an overall correlation of $D_{f}$ with the turbulence level. These are both in the region $2<D_{f}<3$, but larger than the $7 / 3$ limit indicated in [33, and larger than previous findings for singlecomponent fuels with 1-step chemistry in the same flow configuration 29]. It is worth noting that a value of $8 / 3$ was proposed as an upper limit for the fractal dimension by analysing $\mathrm{H}_{2}$-air flame data with detailed chemistry 30 .

The relation of North and Santavica [32] $\left(D=2.05 /\left(u^{\prime} / s_{l}+1\right)+2.35 /\left(s_{l} / u^{\prime}+\right.\right.$ 1)) predicts an upper bound of 2.35 for the fractal dimension with increasing $u^{\prime} / s_{l}$ and this value is lower than that calculated in this study. The values given in Table 3 for the SDR are larger than 3 for all cases indicating a nonfractal or multi-fractal behaviour. The SDR fractal dimension for case B is significantly lower than for case A, and there doesn't appear to be an overall correlation with the turbulence level as in the case for the FSD. This could possibly be because of the multi-fractal behaviour for the SDR which might become stronger with increased turbulence level. Nevertheless, Eq. 12 gives reasonable agreement with the DNS data.

The effect of progress variable definition is also highlighted in Figs. 2 and 3 by showing the results for two additional definitions of $c$ using mass 
fractions. These additional results are shown for selected filter widths. The mass fraction-based $c$ are defined as $c_{O 2}=\left(Y_{O_{2}}-Y_{O_{2} r}\right) /\left(Y_{O_{2} p}-Y_{O_{2} r}\right)$ and $c_{C O}=\left(Y_{C O}-Y_{C O r}\right) /\left(Y_{C O p}-Y_{C O r}\right)$. These species are consumed monotonically in the laminar flame, and are important candidates in the modelling of syngas flames which usually contain large amounts of both $\mathrm{CO}$ and $\mathrm{O}_{2}$. Figure 2 shows that there is little difference in the FSD wrinkling factor variation with filter width compared to that for the temperature based progress variable. In fact, the fractal dimension and inner cut-off scale were found to be almost identical. In the case of the SDR as one observes from Fig. 3 , the wrinkling factors based on the mass fraction progress variables are slightly larger: as a result the inner cut-off scale is slightly lower, however the fractal dimension remains about the same as that for the temperature based progress variable. This implies that the fractal quantities, the inner cut-off and fractal dimension, deduced using temperature based $c$ are quite robust and they can be used to reasonably model FSD and SDR of mass fraction-based $c$. This could be because the overall mass fraction-weighted Lewis number is close to unity.

\subsection{Conditional averages}

In order to assess the performance of the FSD and SDR closures given in section 4 , the conditional averages as obtained from the models are compared to the conditional averages from the DNS data. This is done for cases B and $\mathrm{C}$ only, which are the two highest turbulence level cases and for the smallest and largest filter widths $\left(\Delta^{+}=0.2,5.0\right)$ respectively. Figure 4 shows the conditional average of the filtered FSD, normalised using $\delta_{l}$, obtained using the wrinkling factor models in Eqs. (12) and (14) along with the respective DNS results. Figure 5 shows the conditional average of the filtered SDR both for the fractal-based model in Eq. (12) and for the algebraic models in Eqs. 15 and (17).

In the case of the FSD, for small filter widths the models' predictions are almost identical and in close agreement with the DNS result. For the largest filter width, Eq. (14) under-predicts the filtered FSD. In part, this can be attributed to the lower fractal dimension $\left(D_{f}=2.32\right.$ compared to 2.42$)$ predicted by the empirical relation of North and Santavica [32, however, using the fractal dimension value obtained from the DNS is found to substantially improve this model prediction. It is not easy to know this (right) fractal dimension value a priori and thus one must be cautious in using the fractal attributes deduced using numerical and experimental results of single-component fuels for multi-component fuel flames, specifically with low heating values.

The various SDR model predictions are similar to the DNS values for the smallest filter width i.e. $\Delta^{+}=0.2$ while for the largest filter width there are significant differences as one observes in Fig. 5. The fractal-based model shows the poorest agreement, while the algebraic closures give improved agreement with DNS results. Out of the two algebraic closures in Eqs. 15) and (17), the 
Le-number corrected version shows improved agreement. Nevertheless, this model still shows a substantial deviation from the DNS result across all $\widetilde{c}$. In the context of unstrained flamelet modelling the SDR is a crucial term in the governing equation for the progress variable variance, and it is unclear the extend to which this discrepancy in an actual LES would affect the results for such fuels and a posteriori LES evaluation is required for this. However, there are no experimental data of multi-component fuel combustion for such an evaluation at this time.

\section{Mean reaction rate modelling}

It is worthwhile to explore the performance of some common algebraic reaction rate models originally developed in the RANS context and later extended to LES (showing encouraging results) for the combustion conditions of this study. The specific models of interest are algebraic closures for the filtered reaction rate involving the FSD and SDR. The model involving the SDR was proposed by Bray for RANS [8],

$$
\overline{\dot{w}_{c}}=\frac{2 \bar{\rho} \widetilde{N_{c}}}{2 c_{m}-1}
$$

where a model is required for the SDR $\widetilde{N_{c}}$. This model has been used in the LES context in 54 showing encouraging performance. Based on the results discussed in the previous section, one can choose to use the model given by Eq. (17) which has the smallest error. In order to elucidate the effect of the SDR model on the closure itself, the explicitly filtered value of the SDR is also used. The other filtered reaction rate closure is [55]-58],

$$
\overline{\dot{w}_{c}}=\rho_{r} s_{l} \bar{\Sigma}=\rho_{r} s_{l} \Xi_{\Sigma}
$$

where the filtered FSD is modelled using Eq. (3), with the wrinkling factor model from Eq. (12). These models for the mean reaction rate do not recover the DNS limit as $\Delta^{+} \rightarrow 0$, and bridging functions were proposed in 37 to recover this limit and these functions were obtained explicitly due to the 1-step chemistry scheme employed in those analyses. For flames of multi-component fuels such as the one in this study, it is not quite easy to identify such a bridging function since the heat release occurs over a wider range of progress variable values as seen in Fig. 1, with each species contributing a fraction to the total heat release rate [51,50]. From a practical LES perspective, the limit of zero filter width may not be as severe as it sounds, because the filter width dictated by the numerical grid is typically larger than the laminar flame thickness so that the sub-grid contribution is larger than the resolved contribution implying that the bridging function can be neglected [37.

Figure 6 shows the filtered reaction rate conditionally averaged on a given value of the filtered progress variable, $\dot{w}_{c} \mid \widetilde{c}$, for the models in Eqs. (22) and 
23. These reaction rates are normalised using $\rho_{r}, s_{l}$ and $\delta_{l}$. The model values are shown for two scenarios, i.e., by using the filtered FSD, $\bar{\Sigma}$, or SDR, $\widetilde{N}$, obtained from the DNS and by using their closures. This is done for the largest filter width i.e. $\Delta^{+}=5.0$, where the error is expected to be the largest. Figure 6 shows that for both cases the choice of model for the FSD and SDR significantly affects the mean reaction rate closure. The FSD-based model however appears to be less sensitive than Bray's closure. Both closures give a qualitatively good agreement with the DNS data, however the best agreement (and the one having the lowest error based on $L_{2}$ norm), is Bray's closure with the SDR model given by Eq. 17. This is in contradiction with previous findings in the RANS context, where the same closure (with and without the SDR model) showed a relatively poorer agreement in comparison to the FSD-based closure [48. As a result, the SDR model itself appears to be improving the closure for the filtered reaction rate. Perhaps this would explain the quantitatively good results obtained in the LES of [54] using the Bray closure with the same SDR model i.e. Eq. (17). This result can be explained by examining the variation of the SDR as predicted by Eq. 17 shown in Fig. 5 . This model under-predicts the dissipation rate for relatively low $\widetilde{c}$ values, and over-predicts the dissipation rate for $\widetilde{c}>0.4$ approximately. In these same regions, Bray's closure shows the opposite behaviour as one may see from the results in Fig. 6 (continuous green line). Hence, these two errors cancel out thus improving the predictions of Bray's closure when using the SDR model as specified by Eq. 17

\section{Conclusions}

A multi-component fuel DNS database with complex chemistry is used in order to assess the performance of algebraic-based FSD and SDR models in the context of LES. The fuel composition is akin to the composition of blast furnace gas being increasingly used for power generation. This DNS database has challenging combustion conditions in terms of chemical complexity, flamelet structure, and turbulence-chemistry interactions. Fractal-based models are examined for the FSD and SDR, and some common algebraic closures for the filtered reaction rate involving these two quantities.

The fractal-based models for the FSD wrinkling factor show an overall good agreement with the DNS data for all filter widths tested. The inner cut-off scale is found to be of the order of the laminar flame thickness, i.e., $\eta_{i} \simeq \delta_{l}$, and to about $0.5 \delta_{l}$ with increase in turbulence level while the fractal dimension is observed to increase with increasing turbulence level. This is in accordance with previous DNS studies of single-component fuels with one-step chemistry. However the fractal dimension of the SDR in multi-component fuel flames is found, in general, to be larger than the previously reported values for simple fuel flames. Small changes in the fractal dimension are observed to significantly affect the models' performance. For the fractal-based SDR wrinkling factor models, the inner cut-off scale is found to be very close to the FSD inner cut-off scale but with a larger fractal dimension and this could 
be suggesting that the SDR field has multi-fractal characteristics which need further investigation. However, one must note that the fractal analysis are purely based on the kinematics and does not include the dynamics arising from molecular diffusion which might be more important for the multi-component fuel combustion compared to combustion of simple fuels.

The algebraic SDR models obtained by extending the RANS counterpart are found to perform better in comparison with the wrinkling factor-based closures especially for larger filter widths. The mean reaction rate closure obtained by extending the Bray model for RANS, despite some physical consistency issues in the limit of zero filter width, is observed to give quantitatively good comparison with the DNS data. However, this limit of zero filter width may not be realisable in practical LES and thus this algebraic closure may serve as a good first step in LES of alternative fuel turbulent premixed combustion. All of the observations are based on a priori analysis and one needs to conduct LES of these multi-component fuel flames for further validation which would be explored in the future when some experimental measurements on these flames become available. Nevertheless, a priori testing is a required first step for model development and validation.

Acknowledgements ZMN and NS acknowledge the funding through the Low Carbon Energy University Alliance Programme supported by Tsinghua University, China. ZMN also likes to acknowledge the educational grant through the A.G. Leventis Foundation. This work made use of the facilities of HECToR, the UK's national high-performance computing service, which is provided by UoE HPCx Ltd at the University of Edinburgh, Cray Inc and NAG Ltd, and funded by the Office of Science and Technology through EPSRC's High End Computing Programme. ZMN also acknowledges PRACE for awarding us access to resource Beskow of PDC center for high-performance computing based in Sweden at KTH. 


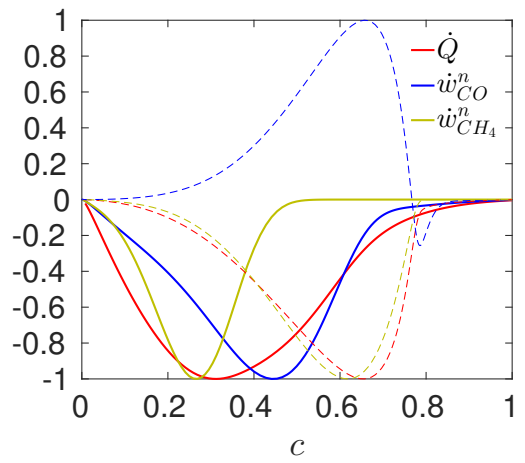

Fig. 1 Variation of normalised reaction rate of fuel species in the progress variable space in laminar flames. The solid lines are for a multi-component fuel considered in the DNS, see Table 2 and the dashed lines are for stoichiometric methane-air flame.

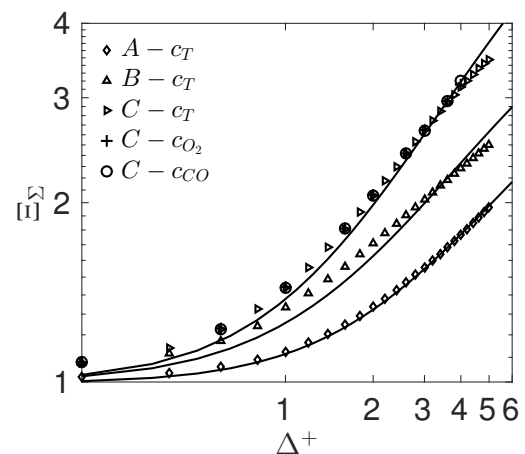

Fig. 2 Variation of FSD wrinkling factor for cases A-C: symbols are DNS data, lines are model values obtained using Eq. 12 (with different model parameters). 


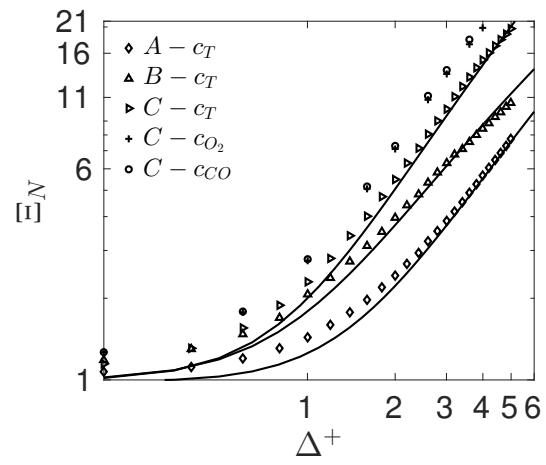

Fig. 3 Variation of SDR wrinkling factor for cases A-C: symbols are DNS data, lines are model values obtained using Eq. 12 (with different model parameters).
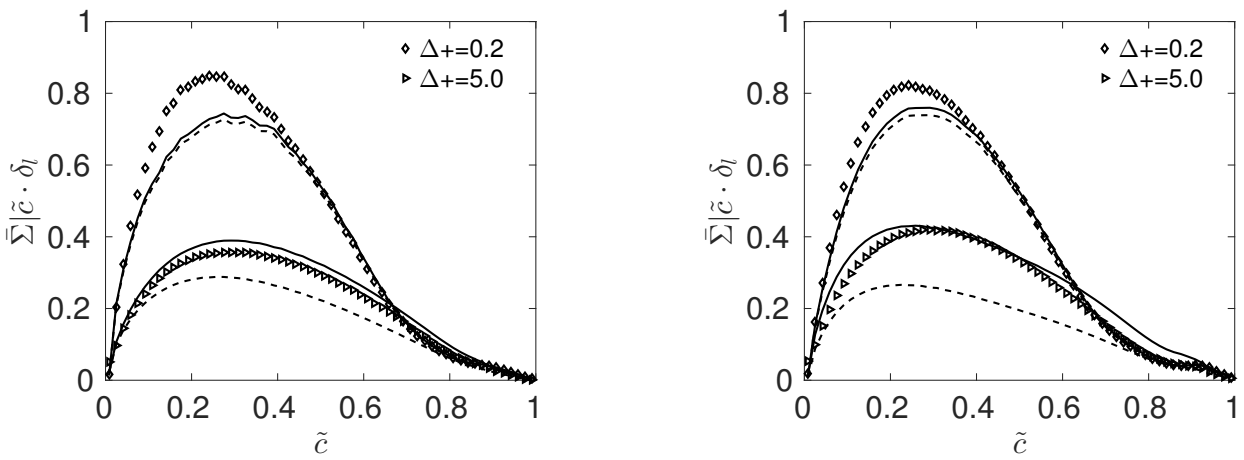

Fig. 4 Conditionally-averaged filtered FSD for case B (left) and case C (right): symbols are DNS data, continuous lines are model predictions using Eq. 12, dashed lines are model prediction using Eq. 14. 

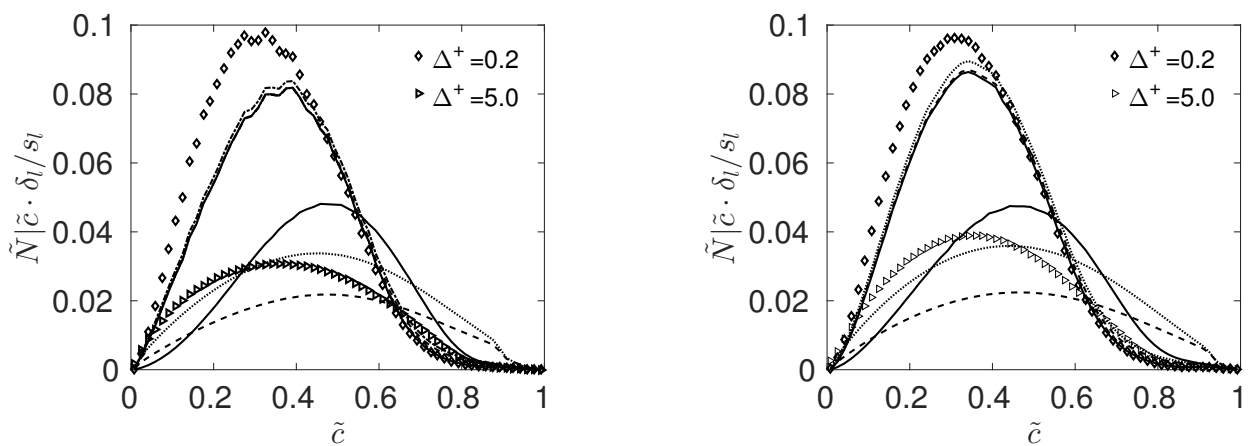

Fig. 5 Conditionally-averaged filtered SDR for case B (left) and case C (right): symbols are DNS data, continuous lines are for Eq. 13], dashed lines are for Eq. 15, dashed-dotted lines are for Eq. 17].
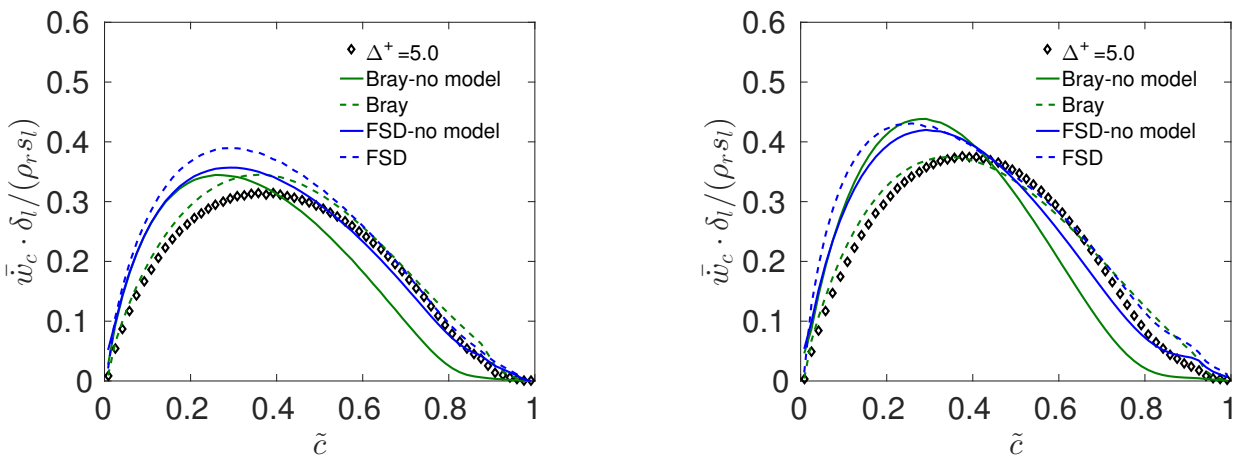

Fig. 6 Conditionally-averaged filtered progress variable reaction rate for case B (left) and case C (right). Symbols are DNS data. 


\begin{tabular}{llllll}
\hline Case & $u_{r m s} / s_{l}$ & $l_{\text {int }} / \delta_{Z}$ & $R e_{t}$ & $D a$ & $K a$ \\
\hline $\mathrm{A}$ & 3.18 & 16.54 & 52.66 & 5.19 & 1.39 \\
$\mathrm{~B}$ & 9.00 & 16.66 & 150.05 & 1.85 & 6.62 \\
$\mathrm{C}$ & 14.04 & 16.43 & 230.69 & 1.17 & 12.97 \\
\hline
\end{tabular}

Table 1: Turbulent flame parameters for the DNS studies.

\begin{tabular}{lllllllll}
\hline Case & $T_{r}(\mathrm{~K})$ & $\phi$ & $p(\mathrm{~atm})$ & $\mathrm{CO}$ & $\mathrm{H}_{2}$ & $\mathrm{H}_{2} \mathrm{O}$ & $\mathrm{CO}_{2}$ & $\mathrm{CH}_{4}$ \\
\hline A-C & 800.0 & 1.0 & 1.0 & 62.687 & 1.881 & 16.000 & 18.806 & 0.627 \\
\hline
\end{tabular}

Table 2: Fuel mixture composition (molar percentages).

\begin{tabular}{lllll}
\hline Case & $\theta_{1}$ & $\theta_{2}$ & $\eta_{i} / \delta_{l}$ & $D_{f}$ \\
\hline A-FSD & 0.10 & 0.24 & 1.045 & 2.43 \\
B-FSD & 0.08 & 0.30 & 0.551 & 2.42 \\
C-FSD & 0.06 & 0.38 & 0.576 & 2.58 \\
A-SDR & 0.02 & 0.45 & 1.032 & 3.30 \\
B-SDR & 0.02 & 0.60 & 0.554 & 3.10 \\
C-SDR & 0.02 & 0.70 & 0.589 & 3.40 \\
\hline
\end{tabular}

Table 3: Optimum model parameters for the FSD and SDR. 


\section{References}

1. Gicquel L.Y.M., Staffelbach G., Poinsot T.: Large eddy simulations of gaseous flames in gas turbine combustion chambers. Prog. En. Combust. Sc. 38, 782-817 (2012)

2. Veynante D., Vervisch L.: Turbulent combustion modelling. Prog. En. Combust. Sc. 28, 193-266 (2002)

3. Mantel T., Borghi R.: A new model of premixed wrinkled flame propagation based on a scalar dissipation equation. Combust. Flame 96, 443-457 (1994)

4. Marble F.E., Broadwell J.E.: The coherent flame model for turbulent chemical reactions. Tech. Rep. TRW-9-PU, Project Squid (1977)

5. Candel S.M., Maistret E., Darabiha N., Poinsot T., Veynante D., Lacas F.: Experimental and numerical studies of turbulent ducted flames. Marble Symposium 209-236 (1988)

6. Pope S.: The evolution of surfaces in turbulence. Int. J. Engng. Sci. 26, 445-469 (1988)

7. Bray K.N.C., Champion M. Libby P.A.: The interaction between turbulence and chemistry in premixed turbulent flames. Turbulent Reactive Flows, pp. 541-563, Lecture notes in engineering, Springer Verlag

8. Bray K.N.C., Moss J.B.: A unified statistical model of the premixed turbulent flame. Acta Astron. 4, 291-319 (1977)

9. Borghi R., Dutoya D.: On the scales of the fluctuations in turbulent combustion. Proc. Combust. Inst. 17, 235-244 (1979)

10. Borghi R.: Turbulent premixed combustion: further discussions on scales of fluctuations. Combust. Flame 80, 304-312 (1990)

11. Mura A., Borghi R.: Towards an extended scalar dissipation equation for turbulent premixed combustion. Combust. Flame 133, 193-196 (2003)

12. Swaminathan N., Grout R.: Interaction of turbulence and scalar fields in premixed flames. Phys. Fluids 18, 045102 (2006)

13. Kolla H., Rogerson J.W., Chakraborty N., Swaminathan N.: Scalar dissipation rate modelling and its validation. Combust. Sci. Tech. 181, 518-535 (2009)

14. Chakraborty N., Swaminathan N.: Influence of the Damkhler number on turbulencescalar interaction in premixed flames I: physical insight Phys. Fluids 19, 045103 (2007)

15. Chakraborty N., Swaminathan N.: Influence of the Damkhler number on turbulencescalar interaction in premixed flames II: model development. Phys. Fluids 19, 045104 (2007)

16. Mura A., Tsuboi K., Hasegawa T.: Modelling of the correlation between velocity and reactive scalar gradients in turbulent premixed flames based on DNS data. Combust. Th. Model. 12, 671-698 (2008)

17. Angelberger C., Veynante D., Egolfopoulos F.: LES of Chemical and Acoustic Forcing of a Premixed Dump Combustor. Flow Turb. Combust. 65, 205-222 (2000)

18. Charlette F., Meneveau C., Veynante D.: A power-law flame wrinkling model for LES of premixed turbulent combustion Part I: non-dynamic formulation and initial tests. Combust. Flame 131, 159-180 (2002).

19. Charlette F., Meneveau C., Veynante D.: A power-law flame wrinkling model for LES of premixed turbulent combustion Part II: dynamic formulation. Combust. Flame 131, 181-197 (2002)

20. Fureby C.: A fractal flame-wrinkling large eddy simulation model for premixed turbulent combustion. Proc. Combust. Inst. 30, 593-601 (2005)

21. Grinstein F.F., Fureby C.: LES studies of the flow in a swirl gas combustor. Proc. Combust. Inst. 2, 1791-1798 (2005)

22. Wang G., Boileau M., Veynante D.: Implementation of a dynamic thickened flame model for large eddy simulations of turbulent premixed combustion. Combust. Flame 11, 2199$2213(2011)$

23. Wang G., Boileau M., Veynante D., Truffin K.: Large eddy simulation of a growing turbulent premixed flame kernel using a dynamic flame surface density model. Combust. Flame 159, 2742-2754 (2012)

24. Volpiani P.S., Schmitt T., Veynante D.: A posteriori tests of a dynamic thickened flame model for large eddy simulations of turbulent premixed combustion. Combust. Flame 174 166-178 (2016) 
25. Mouriaux S., Colin O., Veynatne D.: Adaptation of a dynamic wrinkling model to an engine configuration. Proc. Combust. Inst. 36, 3415-3422 (2017)

26. Peters N.: Turbulent Combustion. Cambridge University Press, Cambridge, UK (2000)

27. Gulder O., Smallwood G.J.: Inner cut-off scale of flame surface wrinkling in turbulent premixed flames. Combust. Flame 103, 107-114 (1995)

28. Knikker R., Veynante D., Meneveau C.: A dynamic flame surface density model for large eddy simulation of turbulent premixed combustion. Phys. Fluids 16, 91-94 (2005)

29. Chakraborty N., Klein M.: A priori direct numerical simulation assessment of algebraic flame surface density models for turbulent premixed flames in the context of large eddy simulation. Phys. Fluids 20, 085108 (2008)

30. O. Chatakonda, E.R. Hawkes, A.J. Aspden, A.R. Kerstein, H. Kolla, J.H. Chen.: On the fractal characteristics of low Damkohler number flames. Combust. Flame 120, 2422-2443 (2013)

31. Roberts W.L., Driscoll J.F., Drake M.C., Goss L.P.: Images of the quenching of a flame by a vortex-to quantify regimes of turbulent combustion. Combust. Flame 94, 58-69 (1993)

32. North G.L., Santavicca D.A.: The fractal nature of turbulent premixed flames. Combust. Sc. Techn. 72, 215-232 (1990)

33. Kerstein A.: Fractal dimension of turbulent premixed flames. Comb. Sc. Techn. 60, 441-445 (1988)

34. Katragadda M., Chakraborty N., Cant. R.S.: Effects of turbulent Reynolds number on the performance of algebraic flame surface density models for Large Eddy Simulation in the thin reaction zones regime: a direct numerical simulation analysis. J. Comb. 794671, (2012)

35. Dunstan T., Minamoto Y., Swaminathan N., Chakraborty N.: Scalar dissipation rate modelling for Large Eddy Simulation of turbulent premixed flames. Proc. Combust. Inst. 34, 1193-1201 (2013)

36. Kolla H., Rogerson J. W., Chakraborty N., Swaminathan N.: Scalar dissipation rate modelling and its validation. Combust. Sci. Technol. 181, 518-535 (2009)

37. Gao Y., Chakraborty N., Swaminathan N.: Algebraic closure of scalar dissipation rate for large eddy simulations of turbulent premixed combustion. Comb. Sc. Tech. 186, 13091337 (2014)

38. Girimaji S., Zhou Y.: Analysis and modelling of sub-grid scalar mixing using numerical data. Phys. Fluids 8, 1224 (1996)

39. Gao Y., Chakraborty N., Swaminathan N.: Dynamic closure of scalar dissipation rate for large eddy simulations of turbulent premixed combustion: a direct numerical simulation analysis. Flow Turb. Combust. 95, 775-802 (2015)

40. Langella I., Swaminathan N., Gao Y., Chakraborty N.: Assessment of dynamic closure for premixed combustion large eddy simulation. Combust. Th. Model. 19, 628-656 (2015)

41. Langella I., Swaminathan N.: Unstrained and strained flamelets for LES of premixed combustion. Combust. Th. Model. 20, 410-440 (2016)

42. Langella I., Swaminathan N., Pitz, R. W.: Application of unstrained flamelet SGS closure for multi-regime premixed combustion. Combust. Flame. 173, 161-178 (2016)

43. Gao Y., Minamoto Y., Tanahashi M., Chakraborty N.: A Priori Assessment of Scalar Dissipation Rate Closure for Large Eddy Simulations of Turbulent Premixed Combustion Using a Detailed Chemistry Direct Numerical Simulation Database. Combust. Sc. Tech. 188, 1398-1423 (2016)

44. Minamoto Y., Fukushima N., Tanahashi M., Miyauchi T., Dunstan T., Swaminathan N.: Effect of flow-geometry on turbulence scalar interaction in premixed flames. Phys. Fluids 23, 125107 (2011)

45. Das A. K., Kumar, K., Sung, C.: Laminar flame speeds of moist syngas mixtures. Combust. Flame 158, 345-353 (2011)

46. Nikolaou Z. M., Chen J. Y., Swaminathan N.: A 5-step reduced mechanism for combustion of $\mathrm{CO} / \mathrm{H} 2 / \mathrm{H} 2 \mathrm{O} / \mathrm{CH} 4 / \mathrm{CO} 2$ mixtures with low hydrogen/methane and high $\mathrm{H} 2 \mathrm{O}$ content. Combust. Flame 160, 56-75 (2013)

47. Singh D., Takayuki N., Saad T., Qiao L.: An experimental and kinetic study of syngas/air combustion at elevated temperatures and the effect of water addition. Fuel 94, 448-456 (2012) 
48. Nikolaou Z.M., Swaminathan N.: Direct numerical simulation of complex fuel combustion with detailed chemistry: physical insight and mean reaction rate modelling. Comb. Sc. Tech. 187, 1759-1789 (2015)

49. Cant R.S. SENGA2 User Guide, CUED/ATHERMO/TR67, September (2012)

50. Nikolaou Z.M., Swaminathan N.: Evaluation of a reduced mechanism for turbulent premixed combustion. Combust. Flame 161, 3085-3099 (2014).

51. Nikolaou Z., Swaminathan N.: A 5-step reduced mechanism for combustion of $\mathrm{CO} / \mathrm{H} 2 / \mathrm{H} 2 \mathrm{O} / \mathrm{CH} 4 / \mathrm{CO} 2$ mixtures with low hydrogen/methane and high $\mathrm{H}_{2} \mathrm{O}$ content. Comb. Flame 160, 56-75 (2013)

52. Peters N.: The turbulent burning velocity for large-scale and small-scale turbulence. J. Fluid Mech. 384, 107-132 (1999)

53. Nikolaou Z., Swaminathan N.: Heat release rate markers for premixed combustion. Comb. Flame 161, 3073-3084 (2014)

54. Butz D., Gao Y., Kempf A.M., Chakraborty N.: Large Eddy Simulations of a turbulent premixed swirl flame using an algebraic scalar dissipation rate closure. Combust. Flame $162,3180-3196(2015)$

55. Cant R.S., Pope S.B., Bray K.N.C.: Modelling of flamelet surface to volume ratio in turbulent premixed combustion. Proc. Combust. Inst. 23, 809-815 (1990)

56. Hawkes E.R., Cant R.S.: A flame surface density approach to large eddy simulation of premixed turbulent combustion. Proc. Combust. Inst. 28, 51-58 (2000)

57. Hawkes E.R., Cant R.S.: Implications of a flame surface density approach to large eddy simulation of premixed turbulent combustion. Combust. Flame 126, 1617-1629 (2001)

58. Chakraborty N., Cant R.S.: Direct numerical simulation analysis of the flame surface density transport equation in the context of large eddy simulation. Proc. Combust. Inst. $32,1445-1453(2009)$ 